\title{
AIMPORTÂNCIA DA ODONTOLOGIA NA ASSISTÊNCIA A INDIVÍDUOS COM DEFEITOS CONGÊNITOS
}

\author{
The importance of Dentistry in assisting individuals with birth defects
}

\author{
Fernanda Diffini Santa Maria ${ }^{a}$, (DBbiana Mello de Oliveira ${ }^{b, c}$, (DLavinia Schuler-Faccini ${ }^{a, b, d}$
}

\section{RESUMO}

Introdução: Defeitos congênitos são alterações estruturais ou funcionais que acontecem durante a vida intra-uterina. O cirurgião-dentista deve reconhecer os defeitos craniofaciais para complementar a caracterização fenotípica e manejá-los junto a uma equipe multiprofissional. A presente revisão tem como objetivo auxiliar o cirurgião-dentista para o diagnóstico desses achados e apresentar quadros sindrômicos tipicamente associados a malformações craniofaciais. Revisão de Literatura: Manifestações craniofaciais de defeitos congênitos são condições que devem ser reconhecidas pelos cirurgiões-dentistas, pois frequentemente estão presentes em sua prática diária, podendo ser este profissional o primeiro a identificar tais achados. Os principais quadros sindrômicos tipicamente associados a micrognatia, fendas orais e displasias esqueléticas com manifestação craniofacial são apresentados, assinalando suas características clínicas e genéticas. Discussão: O cirurgião-dentista deve realizar uma anamnese detalhada incluindo a história familiar, bem como reconhecer as dismorfias tanto clínica quanto radiograficamente, observando o paciente de forma sistêmica. Conclusão: Os profissionais da odontologia devem receber treinamento teórico-prático para o diagnóstico, tratamento e vigilância de indivíduos com defeitos congênitos, seja na avaliação individual ou como parte de uma equipe multiprofissional.

Palavras-chave: Anormalidades congênitas. Odontologia. Anormalidades craniofaciais. Micrognatismo.

\begin{abstract}
Introduction: Birth defects are structural or functional changes that occur during intrauterine life. The dentist must recognize the craniofacial defects, complement the phenotypic characterization and manage them within a multidisciplinary team. The present review aims to assist the dentist to diagnose these findings and present syndromic conditions typically associated with craniofacial malformations. Literature Review: Craniofacial manifestations of birth defects are conditions that must be recognized by dentists, as they are frequently present in their daily practices, and this professional may be the first to identify such findings. The main syndromic clinical pictures typically associated with micrognathia, oral clefts and skeletal dysplasias with craniofacial manifestation are presented, pointing out their clinical and genetic features. Discussion: The dentist must perform a detailed anamnesis including family history, as well as should recognize both clinical and radiographically the dysmorphisms, observing the patient systemically. Conclusion: Dentistry professionals should receive theoretical-practical training for the diagnosis, treatment and surveillance of individuals with congenital defects, either in individual assessment or as part of a multiprofessional team.
\end{abstract}

Keywords: Congenital abnormalities. Dentistry. Craniofacial abnormalities. Micrognathism.

\footnotetext{
'Programa de Pós-graduação em Saúde da Criança e do Adolescente, Universidade Federal do Rio Grande do Sul, Porto Alegre, RS, Brasil. 'Programa de Pós-graduação em Genética e Biologia Molecular, Universidade Federal do Rio Grande do Sul, Porto Alegre, RS, Brasil. 'Irmandade da Santa Casa de Misericórdia de Porto Alegre, Porto Alegre, RS, Brasil. dServiço de Genética Médica, Hospital de Clínicas de Porto Alegre, Porto Alegre, RS, Brasil.
}

Autora de correspondência: Fernanda Diffini Santa Maria - E-mail: nandasantamaria@hotmail.com Data de envio: $31 / 10 / 2020$ | Data de aceite: $01 / 12 / 2020$ 


\section{INTRODUÇÃO}

Defeitos congênitos são alterações estruturais ou funcionais que ocorrem durante a vida intra-uterina, podendo ser identificadas no período pré-natal, ao nascimento ou até mais tarde na vida. São causadas por fatores genéticos, ambientais (teratogênicos) ou mistos (genética e ambiental) ${ }^{1,2}$. Os fatores genéticos correspondem a $45 \%$ das causas de malformação, entretanto a maioria das causas ainda é desconhecida ${ }^{3}$.

De acordo com dados do Estudo Colaborativo Latino Americano de Malformações Congênitas (ECLAMC), a taxa dos defeitos congênitos foi de 4,2\% no Brasil ${ }^{4}$. O Ministério da Saúde descreveu, em 2018, uma prevalência de 0,88\% de defeitos congênitos ao nascimento no Brasil ${ }^{5}$. No Rio Grande do Sul, sua incidência variou de 10 a 15/1.000 recém-nascidos vivos ${ }^{6}$. Já em 2016, a Organização Pan-Americana da Saúde (OPAS) relatou que esses defeitos congênitos são a segunda principal causa de morte em recém-nascidos e crianças menores de cinco anos nas Américas ${ }^{7}$. Os defeitos congênitos são classificados nas seguintes categorias: deformidades, malformações, sequências, disrupções e displasias².

Deformidades ocorrem por atuação de forças mecânicas que distorcem estruturas embrionárias ou fetais previamente normais (por exemplo, face achatada, pés tortos). Tais forças mecânicas podem ser devidas a fatores específicos da mãe (por exemplo, miomas uterinos), da gravidez (por exemplo, gestação múltipla) e/ou do feto (oligodrâmnio, apresentação pélvica) $)^{2,3,8}$.

Disrupção resulta da destruição de estruturas (tecido ou órgão) previamente formadas, em geral ocorrendo numa fase intermediária do desenvolvimento (por exemplo, fendas faciais secundárias a bandas amnióticas) $)^{2,9,10}$.

Displasia é um defeito primário envolvendo uma desorganização das células de um tecido específico (por exemplo, displasias esqueléticas e displasia ectodérmica) 2,9.

Sequência são múltiplas anomalias secundárias a uma anomalia primária. Por exemplo, na sequência de Pierre-Robin há um defeito primário do desenvolvimento da mandíbula, levando a uma mandíbula pequena (micrognatia) que ocasionará uma retração da língua (glossoptose) e disfunção respiratória, com ou sem fechamento inacabado do palato (fenda palatina). Tal sequência poderá levar a problemas na fala e deglutição ${ }^{10}$.

As malformações denotam um problema primário na formação dos tecidos ou órgãos (por exemplo, polidactilia). Malformações podem ocorrer de modo sindrômico, quando associadas a outras malformações ou isoladas. Síndrome é um conjunto de defeitos congênitas que se relacionam patofisiologicamente, entretanto não podem ser explicadas em uma sequência única (por exemplo, Síndrome de Patau) ${ }^{3,10,11}$.

Um defeito congênito poderá causar problemas relevantes não só para o paciente como também para sua família, pois precisarão enfrentar desafios com os cuidados de saúde, e quanto à plena inclusão na sociedade ${ }^{12}$. Portanto os defeitos congênitos são classificados em maiores e menores de acordo com o impacto clínico, social e estético associado².

Anomalias maiores afetam estruturas clinicamente importantes, como por exemplo palato, olhos, cérebro, coração e rins. A maioria das malformações maiores ocorre como defeitos isolados, embora muitas vezes possam ser essenciais para atender aos critérios de diagnóstico de uma síndrome reconhecível. Além disso, podem levar a problemas clínicos e estéticos. Alguns exemplos são fendas orais, síndrome de Down, anencefalia e cardiopatia congênita ${ }^{2,10,13}$.

Anomalias menores são consideradas distúrbios sutis do desenvolvimento e não têm consequências médicas, sociais ou cosméticas graves, mas podem ser bastante frequentes na população em geral como achados isolados. Alguns exemplos são as fossetas, apêndices pré-auriculares e mamilos extranumerários ${ }^{2,10}$. 
Esse artigo tem como objetivo pontuar a importância do profissional da odontologia frente a casos de defeitos craniofaciais congênitos, já que muitas doenças apresentam manifestações craniofaciais e dentárias ${ }^{14,15}$. Tais conhecimentos são fundamentais ao dentista para o diagnóstico dessas condições e adequado encaminhamento do paciente a uma equipe multidisciplinar.

\section{REVISÃO DE LITERATURA}

\section{Malformações frequentes na rotina do cirurgião-dentista}

Fendas orais, macroglossia, agenesias, maloclusões, erupções dentárias precoces ou tardias, malformações dentárias, micrognatia, hipoplasias de esmalte, Displasia Cleidocraniana, Sífilis congênita, sequência de Pierre-Robin e Incontinência Pigmentar são alguns exemplos de malformações craniofaciais congênitas ${ }^{16-20}$. Via de regra essas malformações devem ser reconhecidas pelo dentista.

\section{Fendas orais}

As fissuras orais se dividem em fissura lábio palatina (FLP) e fissura palatina (FP). Os defeitos de fechamento labial ocorrem até a 8. ${ }^{\mathrm{a}}$ semana de desenvolvimento embrionário, enquanto que os de palato ocorrem até a $12 .^{\mathrm{a}}$ semana de vida gestacional ${ }^{21,22}$. As fissuras orais podem ocorrer de forma isolada ou associadas a outros defeitos congênitos ${ }^{23}$, algumas das apresentações sindrômicas mais relevantes e prevalentes são listadas na Tabela 1.

Tabela 1: Principais síndromes genéticas associadas a fendas labiais e/ou palatinas.

\begin{tabular}{|c|c|c|c|}
\hline Síndrome & $\begin{array}{l}\text { Gene ou } \\
\text { locus envolvido }\end{array}$ & Tipo de herança & Outros sinais clínicos \\
\hline Van der Woude 24,25 & IRF6 & $\begin{array}{l}\text { Autossômica } \\
\text { dominante }\end{array}$ & $\begin{array}{l}\text { Fístulas de lábio inferior, geral- } \\
\text { mente bilaterais. }\end{array}$ \\
\hline Stickler ${ }^{25,26}$ & $\begin{array}{l}\text { COL2A1 } \\
\text { COL11A } 1 \\
\text { COL17A2 } \\
\text { COL9A1 } \\
\text { COL9A2 } \\
\text { COL9A3 }\end{array}$ & $\begin{array}{l}\text { Autossômica } \\
\text { dominante e } \\
\text { autossômica } \\
\text { recessiva }\end{array}$ & $\begin{array}{l}\text { Miopia, catarata, descolamento de } \\
\text { retina, perda auditiva condutiva e } \\
\text { neurossensorial, displasia espon- } \\
\text { diloepifisária leve e/ou artrite } \\
\text { precoce. }\end{array}$ \\
\hline $\begin{array}{l}\text { DiGeorge } \\
\text { ou síndrome } \\
\text { Velocardiofacial|25,27 }\end{array}$ & Deleção 22q11.2 & $\begin{array}{l}\text { Autossômica } \\
\text { dominante }\end{array}$ & $\begin{array}{l}\text { Malformações cardíacas congêni- } \\
\text { tas, incompetência velofaríngea, } \\
\text { deficiência imunológica, perda } \\
\text { auditiva neurossensorial e/ou } \\
\text { condutiva. Doenças psiquiátricas e } \\
\text { distúrbios autoimunes. }\end{array}$ \\
\hline Apert ${ }^{25,28}$ & FGFR2 & $\begin{array}{l}\text { Autossômica } \\
\text { dominante }\end{array}$ & $\begin{array}{l}\text { Craniossinostose, retrusão da face } \\
\text { média, sindactilia completa e com- } \\
\text { plexa de mãos e pés. }\end{array}$ \\
\hline
\end{tabular}




\begin{tabular}{|c|c|c|c|}
\hline Síndrome & $\begin{array}{l}\text { Gene ou } \\
\text { locus envolvido }\end{array}$ & Tipo de herança & Outros sinais clínicos \\
\hline $\begin{array}{l}\text { Cornelia de } \\
\text { Lange }^{25,29}\end{array}$ & $\begin{array}{l}\text { NIPBL } \\
\text { RAD21 } \\
\text { SMC3 } \\
\text { HDAC8 } \\
\text { SMC7A }\end{array}$ & $\begin{array}{l}\text { Autossômica } \\
\text { dominante }\end{array}$ & $\begin{array}{l}\text { Restrição de crescimento de início } \\
\text { pré-natal; sinofre, sobrancelhas } \\
\text { altamente arqueadas, hirsutismo } \\
\text { e defeitos de redução de membros } \\
\text { superiores, cílios longos, nariz } \\
\text { curto com narinas antevertidas, } \\
\text { dentes pequenos e espaçados, } \\
\text { microcefalia e defeitos do septo } \\
\text { cardíaco. }\end{array}$ \\
\hline Craniofrontonasal 25 & EFNB1 & $\begin{array}{l}\text { Ligada ao X domi- } \\
\text { nante }\end{array}$ & $\begin{array}{l}\text { Craniossinostose coronal; braqui- } \\
\text { cefalia; hipertelorismo; exotropia; } \\
\text { raiz nasal larga; Ponta nasal bífida; } \\
\text { hérnia diafragmática; hipopla- } \\
\text { sia ou agenesia do corpo caloso. } \\
\text { Expressão mais severa em mulhe- } \\
\text { res que homens. }\end{array}$ \\
\hline Kabuki25 & $K M T 2 D$ & $\begin{array}{l}\text { Autossômica } \\
\text { dominante }\end{array}$ & $\begin{array}{l}\text { Fissuras palpebrais longas com } \\
\text { eversão do terço lateral da pálpe- } \\
\text { bra inferior; sobrancelhas arquea- } \\
\text { das e largas; columela curta com } \\
\text { ponta nasal deprimida; orelhas } \\
\text { grandes, proeminentes ou em } \\
\text { concha; deficiência intelectual leve } \\
\text { a moderada; restrição de cresci- } \\
\text { mento pós-natal; cardiopatias } \\
\text { congênitas; dentes espaçados e } \\
\text { hipodontia; problemas de alimen- } \\
\text { tação e perda auditiva. }\end{array}$ \\
\hline
\end{tabular}

A causa pode ser genética, ambiental ou multifatorial, ou seja, uma combinação de fatores ambientais e genéticos. Os fatores ambientais mais conhecidos são a exposição materna ao tabagismo, álcool e a deficiência de ácido fólico até o primeiro trimestre de gestação $0^{30,31}$. Segundo a Organização Mundial da Saúde (OMS) essas malformações acontecem em uma a cada 650 crianças nascidas no Brasil ${ }^{32}$.

Essas malformações afetam a estética e podem causar problemas auditivos, desnutrição, problemas dentários, bem como dificuldade na fala, possivelmente causando baixa autoestima no paciente ${ }^{32}$.

\section{Micrognatia}

A micrognatia significa mandíbula com tamanho reduzido e posição retrognata em relação à base do crânio ${ }^{33}$. Além disso, pode ser observada de forma isolada ou como características de algumas síndromes como por exemplo Síndrome de Treacher Collins, Síndrome de Franceschetti, Síndrome de Nager, Síndrome de Goldenhar, Sequência de Pierre Robin, Síndrome de Down ou a síndrome de deleção 22q11 ${ }^{34,35}$. Quando há a combinação da micrognatia com glossoptose isso ocasiona no paciente problemas respiratórios e alimentares ${ }^{33}$. 
A sequência de Pierre Robin não é uma síndrome específica e sim um complexo que pode ocorrer de forma isolada ou associada com outras malformações que juntas podem ser relacionadas a uma síndrome específica ${ }^{36}$. Exemplos de síndromes que podem apresentar micrognatia como um de seus achados estão descritas na Tabela 2.

Tabela 2: Síndromes genéticas mais prevalentes e relevantes associadas a micrognatia.

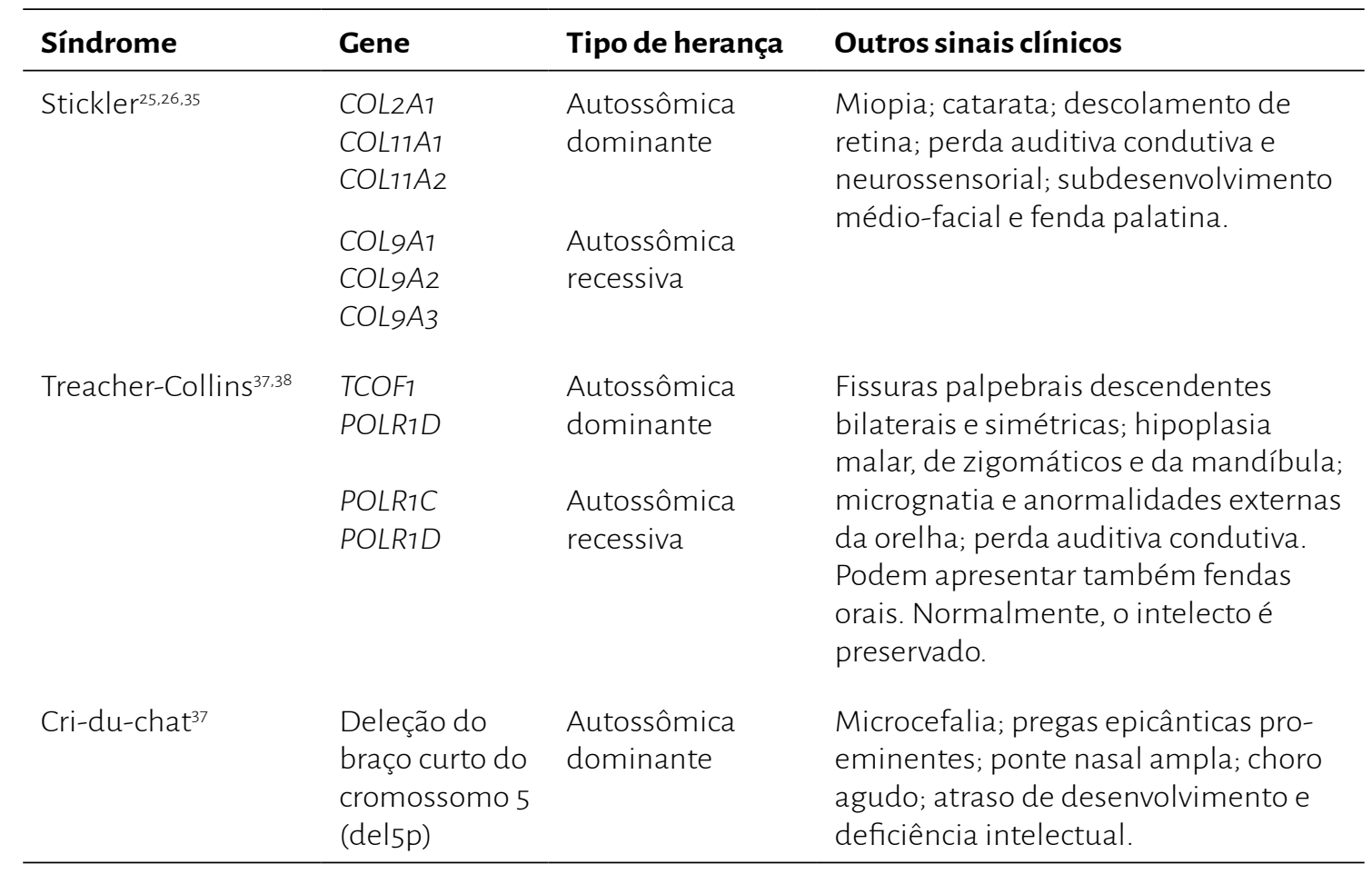

\section{Displasias esqueléticas}

As displasias esqueléticas (DE) também chamadas de displasias ósseas são um conjunto de doenças que apresentam alterações no tecido ósseo e cartilaginoso, que tem como consequência problemas no crescimento. Seu padrão de herança pode ser autossômico recessivo, autossômico dominante, recessivo ligado ao $\mathrm{X}$, dominante ligado ao $\mathrm{X}^{39}$. O número total dessas doenças está em 436 ${ }^{40}$. As DE como grupo apresentam uma frequência de $1 \mathrm{em}$ 3.000 a 5.000 recém-nascidos ${ }^{41}$. Um exemplo é a acondroplasia, que cursa com baixa estatura, macrocefalia com fronte proeminente, ponte nasal baixa, nádegas e abdômen protuberantes, membros com encurtamento proximal (rizomélico) ${ }^{42}$. Sua prevalência é de 1-9 a cada 100.000 nascidos vivos ${ }^{43}$.

Outro exemplo é a Displasia ou Disostose Cleidocraniana, uma síndrome caracterizada pelo atraso no fechamento das suturas cranianas, clavículas hipoplásicas e por alterações na face e dentes. A incidência é de 1-9 em 1.000.000 nascidos vivos e apresenta um padrão de herança autossômico dominante, sendo causada por variantes no gene $R U N X 2(6 \mathrm{p} 21)^{44}$. Alteração na erupção dos dentes permanentes, presença de dentes supranumerários, e atraso na esfoliação dos dentes decíduos são características dessa síndrome ${ }^{45,46}$. Outros exemplos de displasias esqueléticas com acometimento dentário são listados na Tabela 3. 
Tabela 3: Principais displasias esqueléticas com acometimento dentário.

\begin{tabular}{|c|c|c|c|c|}
\hline Síndrome & $\begin{array}{l}\text { Gene } \\
\text { envolvido }\end{array}$ & $\begin{array}{l}\text { Tipo } \\
\text { de herança }\end{array}$ & Alterações dentárias & $\begin{array}{l}\text { Outros sinais } \\
\text { clínicos }\end{array}$ \\
\hline $\begin{array}{l}\text { Displasia clei- } \\
\text { docraniana }{ }^{47}\end{array}$ & $R \cup N X_{2}$ & $\begin{array}{l}\text { Autossômica } \\
\text { dominante }\end{array}$ & $\begin{array}{l}\text { Perda retardada dos } \\
\text { dentes decíduos; apa- } \\
\text { recimento tardio dos } \\
\text { dentes permanen- } \\
\text { tes; dentes cônicos; } \\
\text { supranumerários; má } \\
\text { oclusão. }\end{array}$ & $\begin{array}{l}\text { Clavículas subdesenvol- } \\
\text { vidas ou ausentes; fecha- } \\
\text { mento retardado das sutu- } \\
\text { ras cranianas e fontanelas } \\
\text { amplas; pode haver baixa } \\
\text { estatura; perda auditiva. }\end{array}$ \\
\hline $\begin{array}{l}\text { Osteogenese } \\
\text { imperfeita }{ }^{47,48}\end{array}$ & $\begin{array}{l}\text { COL1A1 } \\
\text { COL1A2 } \\
\text { IFITM5 } \\
\text { CRTAP } \\
\text { SPARC } \\
\text { BMP1 } \\
\text { CREB3L1 } \\
\text { SERPINH1 } \\
\text { P3H1 } \\
\text { WNT1 } \\
\text { SERPINF1 } \\
\text { FKBP10 }\end{array}$ & $\begin{array}{l}\text { Autossômica } \\
\text { recessiva }\end{array}$ & $\begin{array}{l}\text { Coroa dental curta } \\
\text { com exposição den- } \\
\text { tinária ao desgaste } \\
\text { (coroa bulbosa). } \\
\text { Anomalias dentais, } \\
\text { obliteração da polpa } \\
\text { dental, raízes curtas e } \\
\text { delgadas. }\end{array}$ & $\begin{array}{l}\text { Graus variados de fragi- } \\
\text { lidade óssea. Fraturas e } \\
\text { deformidades ósseas de } \\
\text { gravidade e frequência } \\
\text { variáveis, desde formas } \\
\text { letais perinatais até formas } \\
\text { de manifestação e diag- } \\
\text { nóstico na vida adulta. } \\
\text { Pode ocorrer esclera azu- } \\
\text { lada, surdez e fácies com } \\
\text { aspecto triangular. }\end{array}$ \\
\hline $\begin{array}{l}\text { Síndrome de } \\
\text { Braquiolmia- } \\
\text {-amelogenese } \\
\text { imperfeita }^{49}\end{array}$ & ENAM & $\begin{array}{l}\text { Autossômica } \\
\text { recessiva }\end{array}$ & $\begin{array}{l}\text { Amelogênese imper- } \\
\text { feita da dentição pri- } \\
\text { mária e permanente, } \\
\text { com hipomineraliza- } \\
\text { ção do esmalte e des- } \\
\text { coloração dos dentes. } \\
\text { Posição mandibular } \\
\text { retrognática, disgna- } \\
\text { tia vertical e mordida } \\
\text { aberta anterior. }\end{array}$ & $\begin{array}{l}\text { Braquiolmia, platispon- } \\
\text { dilia leve, ilíacos largos, } \\
\text { colos femorais alongados } \\
\text { com coxa valga, escoliose e } \\
\text { baixa estatura com tronco } \\
\text { curto. }\end{array}$ \\
\hline $\begin{array}{l}\text { Displasia } \\
\text { condroec- } \\
\text { todérmica } \\
\text { (Síndrome } \\
\text { de Ellis van } \\
\text { Creveld) }{ }^{47}\end{array}$ & $\begin{array}{l}\text { EVC } \\
\text { EVC2 }\end{array}$ & $\begin{array}{l}\text { Autossômica } \\
\text { recessiva }\end{array}$ & $\begin{array}{l}\text { Fenda alveolar, dentes } \\
\text { neonatais, hipodon- } \\
\text { tia, atraso da erupção } \\
\text { dentária. }\end{array}$ & $\begin{array}{l}\text { Displasia esquelética e } \\
\text { ectodérmica. Tétrade } \\
\text { típica: baixa estatura, poli- } \\
\text { dactilia pós-axial, displasia } \\
\text { ectodérmica e defeitos } \\
\text { cardíacos congênitos. }\end{array}$ \\
\hline $\begin{array}{l}\text { Displasia } \\
\text { Diastrófica }{ }^{47,50}\end{array}$ & $S L C 26 A 2$ & $\begin{array}{l}\text { Autossômica } \\
\text { recessiva }\end{array}$ & $\begin{array}{l}\text { Fenda palatina, palato } \\
\text { alto, micrognatia, } \\
\text { microdontia, apinha- } \\
\text { mento dental. }\end{array}$ & $\begin{array}{l}\text { Estenose laringotraqueal, } \\
\text { calcificação da cartila- } \\
\text { gem costal, cifoescoliose, } \\
\text { encurtamento de ossos } \\
\text { longos, polegar “de caro- } \\
\text { neiro", pés tortos, baixa } \\
\text { estatura. }\end{array}$ \\
\hline $\begin{array}{l}\text { Acondropla- } \\
\operatorname{sia}^{42,43,51}\end{array}$ & FGFR3 & $\begin{array}{l}\text { Autossômica } \\
\text { dominante }\end{array}$ & $\begin{array}{l}\text { Hipoplasia maxilar, } \\
\text { prognatismo mandi- } \\
\text { bular. }\end{array}$ & $\begin{array}{l}\text { Baixa estatura despropor- } \\
\text { cionada, com encurtamento } \\
\text { predominantemente } \\
\text { proximal, macrocefalia com } \\
\text { fronte proeminente e ponte } \\
\text { nasal baixa. }\end{array}$ \\
\hline
\end{tabular}




\section{O papel do cirurgião-dentista frente a um defeito congênito}

Os cirurgiões-dentistas, via de regra, atendem seus pacientes semestralmente, para cuidados preventivos ou semanalmente quando há algum comprometimento maior. Seu campo de trabalho envolve o diagnóstico de alterações na cavidade bucal, facial e cervical ${ }^{52,53}$.

Uma anamnese detalhada, coletando o histórico pessoal e familiar do paciente deve ser realizada. Além disso, um reconhecimento clínico e radiológico de diferentes dismorfias dentárias e craniofaciais se tornam fundamentais para o exame clínico do cirurgião-dentista ${ }^{14,52,54}$. Quando esse profissional se encontra em uma equipe multidisciplinar, uma avaliação sistêmica poderá levar ao diagnóstico de um achado isolado ou sindrômico ${ }^{55}$.

O cirurgião-dentista que observa algum defeito congênito precisa estar preparado para responder às perguntas dos pacientes e saber para onde encaminhá-lo para obter informações ou aconselhamento genético. Quando faz parte de uma equipe multiprofissional, seus achados complementam a caracterização fenotípica e auxiliam no processo diagnóstico. Isso requer uma compreensão básica da genética das doenças humanas, conhecimento dos tipos de testes genéticos disponíveis e sensibilidade às preocupações da família ${ }^{56}$.

Os cirurgiões-dentistas estão em uma posição crucial na identificação de pacientes com doenças genéticas e encaminhamento adequado para centros de atenção a Doenças Raras ${ }^{56}$, e em muitos casos podem ser o primeiro profissional da saúde a identificar determinados defeitos congênitos ${ }^{57}$.

Após o diagnóstico etiológico definitivo de um quadro malformativo, a avaliação odontológica também pode ter papel chave no tratamento e monitorização desta condição. O cirurgião-dentista deverá definir o melhor tratamento odontológico de forma individualizada e depois recomenda-se que seja realizado um acompanhamento permanente da saúde bucal, com a realização de medidas preventivas e reabilitação oral. Isso idealmente deve ser conduzido em uma equipe multiprofissional e com participação do paciente e da família ${ }^{58}$.

\section{DıscUSSÃo}

Essa é a primeira revisão de literatura a enfatizar defeitos congênitos potencialmente identificáveis na prática do cirurgião-dentista e o papel deste profissional frente ao diagnóstico e manejo de tais achados clínicos.

Existem mais de 7.000 diferentes doenças raras em todo o mundo, e muitas dessas envolvem anomalias craniofaciais ${ }^{52}$. Algumas podem permanecer por toda a vida se não corrigidas cirurgicamente e outras podem vir a desaparecer, permanecendo apenas seu comprometimento dentário ${ }^{14,57,59}$.

No caso de tratamento dos pacientes fissurados, o dentista faz parte de uma equipe multidisciplinar, tendo como papel a prevenção, reabilitação e manutenção da saúde bucal do paciente ${ }^{60}$. Já o tratamento de casos com micrognatia na sua forma leve pode ser realizado através de estímulo e sucção no aleitamento materno. Ou também com o uso de aparelhos ortodônticos e/ou ortopédicos funcionais dos maxilares ${ }^{61,62}$. Em casos graves, nos quais o paciente apresenta Sequência de Pierre-Robin, deve ser realizado cirurgia ortognática ou através de alongamento ósseo por estiramento gradual, distração osteogênica da mandíbula com uma equipe multidisciplinar ${ }^{62}$.

Em casos de displasias esqueléticas como a displasia cleidocraniana, o cirurgião-dentista deve estar atento a todas as características clínicas e realizar o tratamento caso a caso. O tratamento terá um protocolo individualizado, pois dependerá da idade do paciente e circunstâncias sistêmicas, bem como da sua adesão ao procedimento indicado ${ }^{63}$. 
O cirurgião-dentista deve ter o conhecimento de conceitos básicos de genética humana e clínica ${ }^{64,65}$. Os cursos de graduação em geral deixam de considerar a genética em sua prática clínica odontológica ${ }^{66}$, porém os conceitos e princípios da genética deveriam passar de um conteúdo teórico para um conhecimento que é aplicado no cuidado de pacientes ${ }^{56}$. Compreender os aspectos genéticos das doenças e gerar novas abordagens para prevenir, diagnosticar e manejar doenças e síndromes criaram novas necessidades para a ciência básica e educação clínica em odontologia. Implicações sociais, éticas e legais novas e desafiadoras estão associadas ao uso de informações genéticas no tratamento e prevenção de doenças ${ }^{56,66}$.

Há evidências crescentes do papel do cirurgião-dentista em reconhecer não apenas distúrbios dentais e orais, mas também indicadores sistêmicos de distúrbios genéticos, tornando esse profissional uma parte integrante da saúde geral e do bem-estar dos pacientes ${ }^{56}$. Esta avaliação poderá subsidiar o encaminhamento a um médico geneticista e assim apoiar o diagnóstico etiológico definitivo, a partir do qual poderá ser iniciado um tratamento específico e aconselhamento genético preciso ${ }^{52}$.

Outra situação recorrente na prática clínica é o exame odontológico como avaliação diagnóstica que apoiará a equipe multiprofissional, após a identificação de achados sistêmicos que possam estar associados a manifestações orais ou craniofaciais. Nestes casos a avaliação é dirigida para achados típicos já identificados em casos anteriormente descritos como agenesias dentais, malformações dentárias ${ }^{18}$, micrognatia ou fendas orais, por exemplo ${ }^{21}$. Como suporte diagnóstico, o cirurgião-dentista pode recorrer a alguns websites amplamente acessíveis tais como Genereviews ${ }^{\circledR 67}$, Online Mendelian Inheritance in Man $\left(\right.$ OMIM $^{47}$, Orphanet $^{49}$ e Phenomizer ${ }^{68}$.

Portanto, a visão de que as atividades do dentista se limitam à manipulação dos dentes está sendo substituída pela visão de que o dentista é um membro importante da equipe de saúde. Existe uma função e responsabilidade ampliadas, e uma parte significativa envolve a atenção a indivíduos com condições genéticas, incluindo o diagnóstico e o processo de aconselhamento genético ${ }^{56}$.

\section{CONCLUSÃo}

O cirurgião-dentista deve reconhecer os aspectos clínicos e radiológicos dos defeitos congênitos. Uma anamnese cuidadosa deve fazer parte da propedêutica, incluindo a revisão de sinais ou sintomas sistêmicos e história familiar.

Assim o cirurgião-dentista terá ferramentas para o diagnóstico de defeitos craniofaciais congênitos ou encaminhamento para confirmação diagnóstica e aconselhamento genético. Os profissionais da odontologia devem receber treinamento teórico e prático para o diagnóstico, tratamento e vigilância de indivíduos com defeitos congênitos, seja na avaliação individual ou como parte de uma equipe multiprofissional.

\section{CONFLITO DE INTERESSES}

Os autores declaram não haver conflito de interesses. 


\section{REFERÊNCIAS}

1. Corsello G, Giuffrè M. Congenital malformations. ] Matern Fetal Neonatal Med. 2012 apr;25(1):25-9.

2. São Paulo (cidade). Secretaria Municipal da Saúde. Coordenação de Epidemiologia e Informação - CEInfo. Declaração de Nascido Vivo - Manual de Anomalias Congênitas. 2. ed. São Paulo: Secretaria Municipal da Saúde; 2012. [acesso em ago 2020]. Disponível em: https://www.prefeitura.sp.gov.br/cidade/secretarias/upload/saude/ arquivos/sinasc/SINASC_ManualAnomaliasCongenitas.pdf.

3. Nussbaum RL, Mclnnes RR, Willard HF. Thompson e Thompson: Genética Médica. 7. ed. Rio de Janeiro: Elsevier Editora Ltda; 2008 .

4. Nazer JH, Cifuentes LO. Malformaciones congénitas en Chile y Latino América: una visión epidemiológica del ECLAMC del período 1995-2008. Rev Méd Chile. 2011;139(1):72-8.

5. Brasil. Ministério da Saúde. DATASUS - Departamento de Informática do SUS; 2008. [acesso em 15 junho 2020]. Disponível em: http://www2.datasus.gov.br/DATASUS/index.php?area=060702.

6. Telles JAB, Schüler-Faccini L. Defeitos congênitos no Rio Grande do Sul: diagnóstico ultrassonográfico pelo estudo morfológico fetal. Rev AMRICS. 2010;54(4):421-6.

7. Organização Pan-Americana de Saúde. OPAS/OMS insta países das Américas a reforçar vigilância de microcefalia e outras anomalias congênitas; 2016. [acesso em 01 ago 2020]. Disponível em: https://www.paho.org.

8. Wenger TL, McDolnal-MacGinn DM, Zackai EH. Genetic of common congenital syndromes of the head and neck. In: Elden LM, Zur KB, editors. Congenital malformation of the head and neck. Springer; 2014 [cited 2020 Aug 20] p. 1-22. Available from: https://doi.org/10.1007/978-1-4419-1714-0_1.

9. Costa AT. O aconselhamento genético como prática clínica: a anencefalia em foco [dissertação]. Rio de Janeiro (R]): Instituto Fernandes Figueira; 2010.

10. Hennekam RC, Biesecker LG, Allanson JE, Hall JG, Optiz JM, Temple IK, et al. Elements of morphology: general terms for congenital anomalies. Am ] Med Genet A. 2013;161(11):2726-33.

11. Kumar V, Abbas AK, Fausto N. Robbins e Cotran. Patologia: bases patológicas das doenças. 7. ed. Rio de Janeiro: Elsevier Editora Ltda; 2004.

12. Horovitz DDG, Llerena JC, Mattos RA. Atenção aos defeitos congênitos no Brasil: panorama atual. Cad Saúde Pública. 2005;21(4):1055-64

13. Rodrigues LS, Lima RHS, Costa LC, Batista RFL. Características das crianças nascidas com malformações congênitas no município de São Luís, Maranhão, 2002-2011. Epidemiol Serv Saúde. 2014;23(2):295-304

14. Kim B], Shin HS, Won CH, Lee JH, Kim KH, Kim MN, et al. Incontinentia pigmenti: clinical observation of 40 Korean cases. J Korean Med Sci. 2006;21(3):474-7.

15. Luo E, Liu H, Zhao Q, Shi B, Chen Q. Dental-craniofacial manifestation and treatment of rare diseases. Int] Oral Sci. 2019;11(1):9.

16. Monlleó IL, Gil-da-Silva-Lopes VL. Anomalias craniofaciais: descrição e avaliação das características gerais da atenção no Sistema Único de Saúde. Cad. Saúde Pública. 2006;22(5):913-22.

17. Bush PG, Williams A]. Incidence of the Robin Anomalad (Pierre Robin syndrome). Br ] Plast Surg. 1983;36:434.

18. Gorlin RJ, Anderson JA. The characteristic dentition of incontinentia pigmenti. ] Pediatr. 1960;57:78-85.

19. Impellizzeri A, Midulla G, Romeo U, La Monaca C, Barbato E, Galluccio G. Delayed eruption of permanent dentition and maxillary contraction in patients with cleidocranial dysplasia: review and report of a family. Int ] Dent. 2018;2018:1-25.

20. Leão ], Gueiros L, Porter S. Oral manifestations of syphilis. Clinics. 2006;61(2):161-6.

21. Holzinger ER, Li Q, Parker MM, Hetmanski JB, Marazita ML, Mangold E, et al. Analysis of sequence data to identify potential risk variants for oral clefts in multiplex families. Mol Genet Genomic Med. 2017;5(5):570-9.

22. Diewert VM. Development of human craniofacial morphology during the late embryonic and early fetal periods. Am ] Orthod. 1985;88:64-76.

23. Wong FK, Hagg U. An update on the aetiology of orofacial clefts. Hong Kong Med ]. 2004;10(5):331-6.

24. Schutte BC, Saal HM, Goudy S, Leslie E. IRF6-Related Disorders. In: Adam MP, Ardinger HH, Pagon RA, Wallace SE, editors. GeneReviews ${ }^{\circledR}$ [Internet]. Seattle (WA): University of Washington; 1993-2021 [updated 2014 ]ul 3; cited 2021 Feb 4]. Available from: https://www.ncbi.nlm.nih.gov/books/NBK1407/.

25. Allam E, Stone C. Cleft lip and palate: etiology, epidemiology, preventive and intervention strategies. Anat Physiol. 2014:4:150.

26. Robin NH, Moran RT, Ala-Kokko L. Stickler Syndrome. In: Adam MP, Ardinger HH, Pagon RA, Wallace SE, editors. GeneReviews ${ }^{\circledR}$ [Internet]. Seattle (WA): University of Washington; 1993-2021 [updated 2017 Mar 16; cited 2021 Feb 4]. Available from: https://www.ncbi.nlm.nih.gov/books/NBK1302/. 
27. McDonald-McGinn DM, Hain HS, Emanuel BS, Zackai EH. 22q11.2 Deletion Syndrome. In: Adam MP, Ardinger $\mathrm{HH}$, Pagon RA, Wallace SE, editors. GeneReviews ${ }^{\circledR}$ [Internet]. Seattle (WA): University of Washington; $1993-2021$ [updated 2020 Feb 27; cited 2021 Feb 4]. Available from: https://www.ncbi.nlm.nih.gov/books/NBK1523/.

28. Wenger TL, Hing AV, Evans KN. Apert Syndrome. In: Adam MP, Ardinger HH, Pagon RA, Wallace SE, editors. GeneReviews $^{\circledR}$ [Internet]. Seattle (WA): University of Washington; 1993-2021. Available from: https://www.ncbi. nlm.nih.gov/books/NBK541728/.

29. Deardorff MA, Noon SE, Krantz ID. Cornelia de Lange Syndrome. In: Adam MP, Ardinger HH, Pagon RA, Wallace SE, editors. GeneReviews ${ }^{\circledR}$ [Internet]. Seattle (WA): University of Washington; 1993-2021 [updated 2020 Oct 15; cited 2021 Feb 4]. Available from: https://www.ncbi.nlm.nih.gov/books/NBK1104/.

30. Vieira AR. Unraveling human cleft lip and palate research. J Dent Res. 2008;87:119-25.

31. Zeiger JS, Beaty TH. Is there a relationship between risk factors for oral clefts? Teratology. 2002;66(3):205-8.

32. Brasil. Ministério da Saúde. Atenção especializada e hospitalar. Fissura labiopalatal; 2013 . [acesso em o1 ago 2020]. Disponível em: https://www.saude.gov.br/atencao-especializada-e-hospitalar/especialidades/cirurgia-plastica-reparadora/fissura-labiopalatal.

33. Scott AR, Tibesar R], Lander TA, Sampson DE, Sidman JD. Mandibular distraction osteogenesis in infants younger than 3 months. Arch Facial Plast Surg. 2011;13(3):173-9.

34. Cohen MM. Robin sequences and complexes: causal heterogeneity and pathogenetic/phenotypic variability. Am ] Med Genet. 1999;84:311-5.

35. Jones K. Smith's recognizable patterns of human malformations. 5. ed. WB Saunders: Philadelphia; 1997.

36. Cohen JrMM. The Robin anomalad: its nonspecificity and associated syndromes. J Oral Surg. 1976;34:587-93.

37. Paladini D. Fetal micrognathia: almost always an ominous finding. Ultrasound Obstet Gynecol. 2010;35(4):377-84.

38. Katsanis SH, Jabs EW. Treacher Collins Syndrome. In: Adam MP, Ardinger HH, Pagon RA, Wallace SE, editors. GeneReviews $^{\circledR}$ [Internet]. Seattle (WA): University of Washington; 1993-2021 [updated 2020 Aug 20; cited 2021 Feb 2]. Available from: https://www.ncbi.nlm.nih.gov/books/NBK1532/.

39. Bonafe L, Cormier-Daire V, Hall C, Lachman R, Mortier G, Mundlos S, et al. Nosology and classification of genetic skeletal disorders: 2015 revision. Am ] Med Genet A. 2015;167(12);2869-92.

40. Mehawej C, Delahodde A, Legeai-Mallet L, Delague V, Kaci N, Desvignes JP, et al. The impairment of MAGMAS function in human is responsible for a severe skeletal dysplasia. PLoS Genet. 2014;10:e1004311.

41. Ascurra M, Herreros MB, Rodriguez S. Displasias oseas: a proposito de cuatro nosologias diferentes. Mem. Inst. Investig Cienc Salud. 2002;1(1):24-7.

42. Shirley ED, Ain MC. Achondroplasia: manifestations and treatment. ] Am Acad Orthop Surg. 2009;17:231-41.

43. Orphanet. Doenças raras. 2020. [acesso em o2 ago 2020]. Disponível em: https://www.orpha.net/consor/cgi-bin/ Disease.php?!ng=PT.

44. Zhang CY, Si Y, Wang XZ, Sun AY, Yan W], Zheng SG. Early dental treatments for patients with Cleidocranial Dysplasia. Chin ] Dent Res. 2015;18:51-7.

45. Golan I, Baumert U, Hrala BP, Müßig D. Dentomaxillofacial variability of cleidocranial dysplasia: clinicoradiological presentation and systematic review. Dentomaxillofac. Radiol. 2003;32(6):347-54.

46. López BSG, Solalinde CO, Ito TK, Carrillo EL, Solalinde EO. Cleidocranial dysplasia: report of a family. J Oral Sci. 2004;46(4):259-66.

47. Online Mendelian Inheritance in Man (Omim). [acesso em 05 ago 2020]. Disponível em: https://omim.org/.

48. Tauer JT, Robinson ME, Rauch F. Osteogenesis imperfecta: new perspectives from clinical and translational research. JBMR Plus. 2019;3:e10174.

49. Orphanet. O portal sobre doenças raras e medicamentos órfãos. [acesso em o2 ago 2020]. Disponível em: https://www.orpha.net/.

50. National Organization for Rare Disorders (NORD). Rare Disease Information. Diastrophic Dysplasia; 2020. [cited 2020 Aug 2]. Available from: https://rarediseases.org/rare-diseases/diastrophic-dysplasia/.

51. Stephen L, Holmes H, Roberts T, Fieggen K, Beighton P. Orthodontic management of achondroplasia in South Africa. S Afr Med ]. 2005;95:588-9.

52. Johnson L, Genco R, Damsky C, Haden NK, Hart S, Hart TC, et al. Genetics and its implications for clinical dental practice and education. J Dent Educ. 2008;72(2 Suppl):86-94.

53. Brasil. Ministério da Saúde. Doenças raras; 2014. [acesso em o2 ago 2020]. Disponível em: http://www.saude. gov.br/saude-de-a-z/doencas-raras.

54. Bailleul-Forestier I, Berdal A, Vinckier F, Ravel T, Fryns ]P, Verloes A, et al. The genetic basis of inherited anomalies of the teeth. Part 2: Syndromes with significant dental involvement. Eur] Med Genet. 2008;5(5):383-408.

55. Hart PS, Hart TC. Invited commentary: The need for human genetics and genomics in dental school curricula. Mol Genet Genomic Med. 2016;4(2):123-25. 
56. Gupta R, Chandra Shekar BR, Goel P, Hongal S, Ganavadiya R. Role of dentist in genetic counseling: a critical appraisal of the current practices and future requirements in Indian scenario. Dent Res ] (Isfahan). 2019;16(3):131-8.

57. Al Khenaizan S. Incontinentia pigmenti. Ann Saudi Med. 2000;20(5-6):424-6.

58. Sandrini FAL, Junior ACC, Beltrão RG, Panarello AF, Robinson WM. Fissuras labiopalatinas em gêmeos: relato de caso. Revista de Cirurgia e Traumatologia Buco-Maxilo-Facial. 2005;5(4):43-8.

59. Al-Zuhaibi S, Ganesh A, Al-Waili A, Al-Azri F, Javad H, Al-Futaisi A. A female child with skin lesions and seizures: case report of incontinentia pigmenti. Sultan Qaboos Univ Med ]. 2009;9(2):157-61.

60. Kuhn VD, Miranda C, Martini DD, Moraes MB, Backes DS, Martins JS, et al. Fissuras labiopalatais: revisão de literatura. Disciplinarum Scientia. 2012;13(2):237-45.

61. Strohecker B, Lahey D. Mandibular elongation by bone distraction: treatment for mandibular hypoplasia with Robin Sequence. Plast Surg Nurs Spring. 1997;17(1):8-10.

62. Vaccari-Mazzetti MP, Kobata CT, Brock RS. Distração osteogênica e tratamento ortopédico na Síndrome de Pierre Robin. Arq Catarin Med. 2009;38(1):52-4.

63. Farrow E, Nicot R, Wiss A, Laborde A, Ferri ]. Cleidocranial dysplasia: a review of clinical, radiological, genetic implications and a guidelines proposal. J Craniofac Surg. 2018;29(2):382-9.

64. Regier DS, Hart TC. Genetics: the future is now with interprofessional collaboration. Dent Clin North Am. 2016;60(4):943-9.

65. Meyer DM, Kaste LM, Lituri KM, Tomar S, Fox CH, Petersen PE. Policy development fosters collaborative practice. Dent Clin N Am. 2016;60(4):921-42.

66. Casado PL, Quinelato V, Cataldo P, Prazeres ], Campello M, Bonato LL, et al. Dental genetics in Brazil: Where we are. Mol Genet Genomic Med. 2018;6:689-701.

67. Adam MP, Ardinger HH, Pagon RA, Wallace SE, editors. GeneReviews ${ }^{\circledR}$ [Internet]. Seattle (WA): University of Washington; 1993-2021 [cited 2021 Feb 4]. Available from: https://www.ncbi.nlm.nih.gov/books/NBK1116/.

68. Phenomizer. [acesso em 02 ago 2020]. Disponível em: http://compbio.charite.de/phenomizer/. 